\title{
The External Shock Model of Gamma-Ray Bursts: Three Predictions and a Paradox Resolved
}

\author{
Charles D. Dermer ${ }^{1}$, Markus Böttcher ${ }^{2,1}$, and James Chiang $3,1,4$
}

\begin{abstract}
In the external shock model, gamma-ray burst (GRB) emissions are produced by the energization and deceleration of a thin relativistic blast wave due to its interactions with the circumburst medium (CBM). We study the physical properties of an analytic function which describes temporally-evolving GRB spectra in the limit of a smooth CBM with density $n(x) \propto x^{-\eta}$, where $x$ is the radial coordinate. The hard-to-soft spectral evolution and the intensity-hardness correlation of GRB peaks are reproduced. We predict that (1) GRB peaks are aligned at high photon energies and lag at low energies according to a simple rule; that (2) temporal indices at the leading edge of a GRB peak display a well-defined shift with photon energy; and that (3) the change in the spectral index values between the leading and trailing edges of a GRB peak decreases at higher photon energies. The reason that GRBs are usually detected with $\nu F_{\nu}$ peaks in the $50 \mathrm{keV}$ - several $\mathrm{MeV}$ range for detectors which trigger on peak flux over a fixed time interval is shown to be a consequence of the inverse correlation of peak flux and duration of the radiation emitted by decelerating blast waves.
\end{abstract}

Subject headings: gamma rays: bursts — gamma rays: theory — radiation mechanisms: nonthermal

\section{Introduction}

The Beppo-SAX results have revolutionized our understanding of GRBs by opening a window on X-ray, optical, and radio afterglows which are delayed from the prompt X-ray and $\gamma$-ray emissions by several hours and more (e.g., Costa et al. 1997; Feroci et al. 1998; Piro et al. 1998; van Paradijs et al. 1997; Frail 1998). The decaying power-law long-wavelength afterglows are compellingly explained as the emissions from a relativistic blast wave which decelerates and radiates through the process of sweeping up material from a uniform CBM (e.g., Paczyński \&

\footnotetext{
${ }^{1}$ E. O. Hulburt Center for Space Research, Code 7653, Naval Research Laboratory, Washington, DC 20375-5352

${ }^{2}$ Department of Space Physics and Astronomy, Rice University, Houston, TX 77005-1892

${ }^{3}$ JILA, University of Colorado, Boulder, CO 80309-0440

${ }^{4}$ NRL/NRC Resident Research Asscociate
} 
Rhoads 1993; Katz 1994; Mészáros \& Rees 1997; Wijers, Rees, \& Mészáros 1997; Tavani 1997). Afterglow behaviors more complicated than simple power laws may involve effects of extinction, self-absorption, scintillation, and CBM structure.

Mészáros \& Rees (1993) proposed that the energy of a fireball's blast wave could be efficiently converted into radiation during the prompt gamma-ray luminous phase of a GRB through the interaction of a blast wave with the CBM. In the external shock model, all GRB emissions are

due to the effects of a single thin blast wave which interacts with the CBM. Because the directed kinetic energy of the blast wave is converted into nonthermal particle energy by sweeping up material from the CBM, the emitted radiation depends crucially on the density distribution of the CBM in the blast wave's path. Smoother GRB time profiles therefore represent more uniform CBMs, at least within the Doppler cone from which most of the detected GRB emissions originate. Conversely, erratic and spiky GRB time profiles represent blast-wave deceleration in highly textured CBMs (Dermer \& Mitman 1999).

In a recent paper (Dermer, Chiang, \& Böttcher 1999; hereafter DCB), we proposed a parametric description of the radiation observed from a spherically expanding blast wave which decelerates and is energized by sweeping up material from a smooth CBM. By "smooth" we mean that the CBM density distribution is adequately represented by the expression $n(x)=n_{0} x^{-\eta}$, where $x$ is the radial coordinate. In this limit, the deceleration of the blast wave produces a time profile which mimics the so-called Fast Rise, Exponential Decay (FRED) GRB light curves. Our ansatz is that a smooth CBM produces an ideal FRED light curve. Conversely, a GRB which exhibits the classic FRED-type profile results from a fireball embedded within and expanding into a smooth CBM.

In this Letter, we make three quantitative predictions that can be tested using BATSE and Beppo-SAX data from bright FRED-type GRB time profiles, though the predictions would be most thoroughly tested with a GRB telescope which is much more sensitive than BATSE and measures prompt GRB emission in the range between $\approx 1 \mathrm{keV}$ and several $\mathrm{MeV}$. In Section 2 , we describe the model equations derived in DCB. Spectral and temporal predictions are given analytically and described graphically for a model fireball with $\Gamma_{0}=300$. In Section 3 we show that the reason that GRBs are usually observed with $\nu F_{\nu}$ peaks in the $\sim 50 \mathrm{keV}$-several $\mathrm{MeV}$ range is understood when account is taken of blast-wave physics and the triggering properties of burst detectors.

\section{Analytic Description and Predictions of the Blast Wave Model}

In DBC, a parametrization of the nonthermal synchrotron emission from a decelerating blast wave observed at time $t$ and photon energy $\epsilon=h \nu / m_{e} c^{2}$ was proposed. The function is based on the analytic approach of Dermer \& Chiang (1998) and the numerical results of Chiang \& Dermer (1999), which were in turn based upon the blast-wave physics developed by, e.g., Blandford \& 
McKee (1976), Rees \& Mészáros (1992), Piran \& Shemi (1993), Mészáros \& Rees (1993), Mészáros, Laguna, \& Rees (1993), Waxman (1997), and Vietri (1997).

The deceleration of the relativistic blast wave is assumed to follow the expression $\Gamma(x)=\Gamma_{0}$ when $x \leq x_{d}$, and $\Gamma(x)=\Gamma_{0}\left(x / x_{d}\right)^{-g}$ when $x_{d} \leq x \leq x_{d} \Gamma_{0}^{1 / g}$. Here $\Gamma_{0}$ is the initial bulk Lorentz factor of the blast wave and the decleration radius $x_{d}=2.6 \times 10^{16}\left[(1-\eta / 3) E_{54} /\left(n_{2} \Gamma_{300}^{2}\right)\right]^{1 / 3}$ $\mathrm{cm}$. In this relation, the burst source emits $\partial E / \partial \Omega=10^{54} E_{54} /(4 \pi) \mathrm{ergs} \mathrm{sr}^{-1}, \Gamma_{0} \equiv 300 \Gamma_{300}$, and $n_{2}=n_{0} /\left(10^{2} \mathrm{~cm}^{-3}\right)$. For simplicity, we consider only spherically symmetric blast waves. The parameter $g$ specifies the radiative regime, and $g \rightarrow(3-\eta) / 2$ and $g \rightarrow 3-\eta$ in the adiabatic and radiative limits, respectively. The deceleration time scale $t_{d}=9.7(1+z)\left[(1-\eta / 3) E_{54} /\left(n_{2} \Gamma_{300}^{8}\right)\right]^{1 / 3}$ s.

The function proposed in DBC to model the blast-wave radiation has the broken-power law form

$$
P(\epsilon, t)=4 \pi d_{L}^{2} \nu F_{\nu}=\frac{(1+v / \delta) P_{p}(t)}{\left[\epsilon / \epsilon_{p}(t)\right]^{-v}+(v / \delta)\left[\epsilon / \epsilon_{p}(t)\right]^{\delta}},
$$

where $v$ and $\delta$ are the $\nu F_{\nu}$ spectral indices at energies below and above the temporally-evolving break energy $\epsilon_{p}(t)$, respectively. Expressions for $\epsilon_{p}(t)=\mathcal{E}_{0}\left[\Gamma(x) / \Gamma_{0}\right]^{4}\left(x / x_{d}\right)^{-\eta / 2}$ and the time-varying amplitude $P_{p}(t)=\Pi_{0}\left[\Gamma(x) / \Gamma_{0}\right]^{4}\left(x / x_{d}\right)^{2-\eta}$ are given in DBC by eqs.(9) and (16), respectively. Eq. (1) depends on the specification of the nine parameters listed in Table 1. The equipartion parameter $q=\left[\xi_{H}(r / 4)\right]^{1 / 2} \xi_{e}^{2}$, where $r$ is the compression ratio and $\xi_{H}$ and $\xi_{e}$ are the magnetic field and electron equipartition values, respectively (see eqs. [7] and [8] in DCB). The parameters $v, \delta, g, \eta$, and $q$ are assumed to be independent of time. This assumption is obviously not true in general, but is realized in the limit that high-quality broadband measurements from a bright FRED-type GRB are made over a sufficiently short observing times $\delta t$ so that radiative-cooling and magnetic field-evolution time scales in the comoving frame are $\gg \Gamma \delta t /(1+z)$.

The thick curves in Fig. 1 show time profiles calculated at various observing energies $\epsilon$, using the equations of the previous section. The thin curves show spectral indices calculated between $\epsilon$ and $2 \epsilon$. Here we show results for fireballs with $\Gamma_{0}=300$ which are located at redshift $z=1$; other parameters are listed in Table 1 . In this example, $t_{d}=9.6(1+z) \mathrm{s}$ and $\mathcal{E}_{0}=2.43 /(1+z)$. The model GRB light curves shown in Fig. 1 display a rapid rise followed by a gentler decay which approaches a power-law afterglow behavior $\propto t^{-1.52}$ (see eq. [22] in DCB) at late times $t \gg t_{d}$. The overall shape of the model light curves shown in Fig. 1 resembles the characteristic FRED-type GRB light curve (e.g., Fishman \& Meegan 1995). The model GRB represents a very bright BATSE GRB if located at $z=1$, but would fall below BATSE detectablity if located at $z \gtrsim 3$.

The analytic approximation used for the evolution of $\Gamma(x)$ gives spectral index curves which are constant when $t \leq t_{d}$. At later times, the blast-wave deceleration means that an observer measuring a photon spectrum over a fixed range of energies will sample photons which are produced at progressively higher energies in the blast wave's frame of reference. Because a nonthermal synchrotron spectrum from a power-law distribution of electrons with a low energy 
cutoff is very hard at low energies and softens at higher energies, blast-wave deceleration produces the hard-to-soft spectral evolution observed in many GRB time profiles (Norris et al. 1986). If the observer is monitoring a GRB at $\epsilon \gtrsim \mathcal{E}_{0}$, the flux is brightest when $t \approx t_{d}$. As can be seen from Fig. 1, the spectrum remains hard until $t \gtrsim t_{d}$, after which the blast wave begins to decelerate and the spectrum softens. Thus the spectrum is hardest when it is most intense, accounting for the hardness-intensity correlation observed in GRB light curves (Golenetskii et al. 1983). If the blast wave produces short time scale variability by interacting with inhomogeneities in the CBM (Dermer \& Mitman 1999), then the individual pulses in GRB profiles would likewise exhibit spectral hardening and subsequent softening, as generally observed in well-defined pulses of GRB light curves (Ford et al. 1995; Crider et al. 1998). The qualitative ability of the blast wave model to explain these empirical trends has been pointed out previously by Panaitescu \& Mészáros (1998).

We now propose three quantitative predictions to test the validity of this model. As is evident from Fig. 1, the peak flux shifts to later times at lower photon energies. The peak times are given analytically by the expression

$$
t_{p}(\epsilon)=t_{d} \max \left\{1,(1+2 g)^{-1}\left[\left(\frac{\epsilon}{\mathcal{E}_{0}}\right)^{-\frac{2 g+1}{4 g+\eta / 2}}+2 g\right]\right\}
$$

(see also Chiang 1998). By plotting the times of a well-defined peak for a GRB measured over a large energy range, one tests the external shock model and constrains values of $\mathcal{E}_{0}$ and the index $(2 g+1) /(4 g+\eta / 2)$. The peak shifting will be more pronounced in GRBs with larger values of $\mathcal{E}_{0}$.

Our second prediction is that the temporal indices of a pulse profile vary with photon energy according to the relation $\chi(\epsilon ; t)=\partial \ln P(\epsilon, t) / \partial \ln t$. This yields a complicated analytic expression from eq. (1), though it is easily calculated numerically. When $t<t_{d}$, however, the analytic form gives

$$
P(\epsilon, t)=\Pi_{0} \times \begin{cases}\left(1+\frac{v}{\delta}\right)\left(\frac{\epsilon}{\mathcal{E}_{0}}\right)^{-v}\left(t / t_{d}\right)^{2-\eta+\eta v / 2} & \text { for } \epsilon \ll \mathcal{E}_{0}, \text { and } \\ \left(1+\frac{\delta}{v}\right)\left(\frac{\epsilon}{\mathcal{E}_{0}}\right)^{-\delta}\left(t / t_{d}\right)^{2-\eta-\eta \delta / 2} & \text { for } \epsilon \gg \mathcal{E}_{0} .\end{cases}
$$

Thus we predict that the temporal index $\chi$ changes from $2-\eta(1-v / 2)$ at low energies to $2-\eta(1+\delta / 2)$ at high energies. For a uniform CBM, there is no shift and the temporal index $\chi=2$. When $\eta \neq 0$, the relation between the temporal and spectral indices indicated by eq. (3) implies the value of $\eta$.

As can also be seen from Fig. 1, the spectral index is hardest at the leading edge of a GRB peak and softens at the trailing edge, with the change in the values of the spectral index decreasing towards higher photon energies. This prediction can be made quantitative by taking the derivative of eq. (1), giving the result

$$
\alpha(\epsilon, t)=\frac{\partial \ln P(\epsilon, t)}{\partial \ln \epsilon}=\frac{v\left(y^{-v}-y^{\delta}\right)}{y^{-v}+(v / \delta) y^{\delta}}
$$


for the $\nu F_{\nu}$ spectral index, where $y=\epsilon / \epsilon_{p}(t)$. The external shock model can be tested by plotting the energy-dependent variation of the spectral index across the peak of a GRB from eq. (4).

Although the above equations provide a simple analytic characterization of the predictions of the external shock model, they depend crucially on the $\Gamma(x)$-prescription noted above. Moreover, electron cooling is not taken into account in the analytic model. We used a numerical simulation code (Chiang \& Dermer 1999) to determine the reliability of the analytic predictions. The results are shown in Fig. 2 using the parameters of Table 1. Because the radiative regime $g$ in the simulation depends on the magnetic field $H(\mathrm{G})$, the value of $\xi_{H}=H^{2} /\left[32 \pi m_{p} c^{2} n_{0}(r / 4)\right]$ was adjusted until the bulk Lorentz factor approached the asymptotic behavior $\Gamma \propto x^{-2}$ in the deceleration phase. This occurred for $\xi_{H} \cong 3 \times 10^{-5}$. The injection spectrum of electrons is chosen $\propto \gamma^{-3.4}$ in order to give an uncooled spectrum with $\delta=0.2$. Electron cooling is seen to be important at high photon energies. The peak of the light curves at large photon energies occurs at $\approx t_{d} / 2$, with a peak flux $\approx 2 \times$ greater than the analytic estimate. We find that the qualitative trends of the predictions described above are reproduced in the detailed calculation. The analytic model can be used to confine the parameter range, but detailed fitting to data should employ the more accurate numerical simulations.

\section{Resolution of the Relativistic Beaming $/ \nu F_{\nu}$ Peak Energy Paradox}

Even if cosmic fireballs were produced with a narrow range of values of $\Gamma_{0}$, their $\nu F_{\nu}$ peaks would be distributed over a wide range of photon energies since $\mathcal{E}_{0} \propto \Gamma_{0}^{4}$. It has therefore been something of a mystery to understand why GRBs are usually detected with $\nu F_{\nu}$ peaks in a narrow range of photon energies between $\sim 50 \mathrm{keV}$ and $\sim 1 \mathrm{MeV}$ (see, e.g., Malozzi et al. 1995; Piran \& Narayan 1996) without requiring fine-tuning of the parameters. Brainerd's (1994) Compton attenuation model, for example, has been advanced to specifically address this puzzle. By contrast to GRBs, the nonthermal emissions in blazars are also thought to originate from relativistic outflows, yet they have $\nu F_{\nu}$ peaks in both their synchrotron and Compton components which range over three orders-of-magnitude or more in photon energy (e.g., von Montigny et al. 1995).

Using the spectral form proposed in DCB, we show how this paradox is resolved for a uniform CBM (the generalization to $\eta \neq 0$ is straightforward). Suppose an instrument triggers on peak flux measured over the time scale $\Delta t$ and over a narrow range of photon energies centered at $\epsilon_{d}$. When the mean duration $t_{p}\left(\epsilon_{d}\right)$ of the fireball measured at $\epsilon_{d}$ is longer than $\Delta t$, then the detector triggers on peak flux. From the expressions for $\epsilon_{p}(t)$ and $P_{p}(t)$, we find that fireballs with $\Gamma_{0}=\bar{\Gamma}_{0} \cong 240\left[(1+z) \epsilon_{d} /\left(n_{2}^{3 / 8} q_{-3}\right)\right]^{1 / 4}$ are observed with the peak of their $\nu F_{\nu}$ spectrum at photon energy $\epsilon_{d}$ at the moment when the received bolometric power is greatest (i.e., when $\left.t=t_{d}\right)$. Dirtier fireballs with $\Gamma_{0}<\bar{\Gamma}_{0}$ produce a peak flux at $\epsilon_{d}$ which declines according to the relation $P_{p}\left(\epsilon_{d}, t_{p}\right) \propto \Gamma_{0}^{(4 \delta+8 / 3)}$, as can be derived by inserting eq. (2) into eq. (1) in the limit $\epsilon_{d} \gg \mathcal{E}_{0}$ (see also eq. [19] in DCB). Because $\delta>0$, the peak flux therefore rapidly decreases with 
decreasing $\Gamma_{0}$. Cleaner fireballs with $\Gamma_{0}>\bar{\Gamma}_{0}$ are also more difficult to detect because in this case, $P_{p}\left(\epsilon_{d}, t_{p}\right) \propto \Gamma_{0}^{2 g^{-1}-4 / 3}$ (cf. eq. [20] in DCB). In order to be observed at all, blast waves cannot be perfectly adiabatic, so that $g>3 / 2$ and the peak flux detected with a GRB instrument decreases with increasing $\Gamma_{0}$.

Cleaner fireballs are more difficult to detect than indicated above, because when $t_{d} \ll \Delta t$, the detector triggers on fluence rather than flux. The fluence $F$ decreases with increasing $\Gamma_{0}$ according $F \propto t_{p} P_{p}\left(\epsilon_{d}, t_{p}\right) \propto \Gamma_{0}^{g^{-1}-2 / 3} \times \Gamma_{0}^{2 g^{-1}-4 / 3}$, using eq. (2) and the expression for $\epsilon_{p}(t)$ with $\eta=0$. Because of the emission properties of blast waves, detectors which trigger on peak flux over a fixed time window will therefore be most sensitive to fireballs which have $\nu F_{\nu}$ peaks in the energy range of the detector. Hence the discovery of new classes of clean and dirty fireballs must consider varying time windows and energy ranges in the triggering criteria of a detector, and must also contend with the levels of background radiation as discussed in DCB.

In separate work (Böttcher \& Dermer 1999), we model the triggering properties of GRB detectors and show that compared to the BATSE GRB trigger rate, the dirty fireball rate is poorly known due to selection biases against their detection. Observational analyses of Ariel $V$ X-ray data (Grindlay 1999; see discussion in DCB) provides the strongest available limit on the frequency of dirty fireballs. By contrast, the relative rate of clean fireballs is strongly constrained in the analysis of Böttcher \& Dermer (1999) unless there is a strong anti-correlation between $\Gamma_{0}$ and $q$.

In summary, we have used a simple analytic description of the external shock model of GRBs to pose three predictions which can be tested by fitting eqs. (2)-(4) to high quality data obtained with a GRB telescope sensitive in the $\approx 1 \mathrm{keV}$ - several MeV range. Although we have employed a simple mathematical parametrization, detailed fits to data should use numerical simulations of the decelerating blast wave (e.g., Chiang \& Dermer 1999, Panaitescu \& Meszaros 1998). We have also explained why detectors which trigger on peak flux are most sensitive to fireballs which produce $\nu F_{\nu}$ peaks in the $50 \mathrm{keV}$ - several $\mathrm{MeV}$ range, resolving a long-standing puzzle in relativistic beaming models of GRBs.

We thank the referee for comments. The work of CD and MB was partially supported by the Compton Gamma Ray Observatory Guest Investigator Program. The work of JC was performed while he held a National Research Council - Naval Research Laboratory Associateship. CD acknowledges support of the Office of Naval Research.

\section{REFERENCES}

Blandford, R. D., \& McKee, C. F. 1976, Phys. Fluids, 19,1130

Böttcher, M., \& Dermer, C. D. 1999, ApJ, submitted (astro-ph/9812059)

Brainerd, J. J. 1994, ApJ, 428, 21 
Table 1: Standard Parameters for Model of Evolving GRB Spectra

\begin{tabular}{cll}
\hline \hline Parameter & Standard Value & Description \\
& & \\
\hline$\Gamma_{0}$ & 300 & baryon-loading parameter \\
$q$ & $10^{-3}$ & equipartition term \\
$g$ & 2 & index of $\Gamma$ evolution \\
$\partial E_{0} / \partial \Omega$ & $10^{54} E_{54} /(4 \pi)$ & total fireball energy released per unit solid angle, ergs sr \\
$v$ & $4 / 3$ & spectral index of rising portion of $\nu L_{\nu}$ spectrum \\
$\delta$ & 0.2 & spectral index of falling portion of $\nu L_{\nu}$ spectrum \\
$z$ & 1 & cosmological redshift \\
$n_{0}$ & $10^{2} \mathrm{~cm}^{-3}$ & density at $x_{d}$ \\
$\eta$ & 0 & index of density distribution \\
\hline
\end{tabular}

Chiang, J. 1998, ApJ, 508, 752

Chiang, J., \& Dermer, C. D. 1999, ApJ, in press astro-ph/9803339)

Costa, E., et al. 1997, Nature, 387, 783

Crider, A., et al. 1998, in preparation

Dermer, C. D., Chiang, J., \& Böttcher, M., 1999, ApJ, in press (DCB; astro-ph/9804174)

Dermer, C. D., \& Chiang, J. 1998, New Astronomy, 3, 157

Dermer, C. D., \& Mitman, K. E. 1999, ApJL, 513, 000

Fenimore, E. E., Madras, C., \& Nayakshin, S. 1996, ApJ, 473, 998

Feroci, M. et al. 1998, A\&A, 323, L29

Fishman, G. J., \& Meegan, C. A. 1995, ARAA, 33, 415

Ford, L. A., et al. 1995, ApJ, 439, 307

Frail, D. 1998, in the Fourth Huntsville Gamma-Ray Burst Symposium, ed. C. A. Meegan, R. D. Preece, \& T. M. Koshut (AIP: New York),

Golenetskii, S. V. et al. 1983, Nature, 306, 451

Grindlay, J. E. 1999, ApJ, in press astro-ph/9808242)

Katz, J. I. 1994, ApJ, 422, 248

Mallozzi, R. S. et al. 1998, ApJ, 454, 597

Mészáros, P., \& Rees, M. J. 1993b, ApJ, 405, 278

Mészáros, P., \& Rees, M. J. 1997, ApJ, 476, 232

Mészáros, P., Laguna, P., \& Rees, M. J. 1993, ApJ, 415, 181

Norris, J., et al. 1986, ApJ, 301, 213 
Paczyński, B., \& Rhoads, J. 1993, ApJ, 418, L5

Panaitescu, A., \& Mészáros, P. 1998, ApJ, 492, 683

Piro, L., et al. 1998, A\&A, 331, L41

Piran, T., \& Narayan, R. in Gamma-Ray Bursts: Third Huntsville Symposium, eds. C. Kouveliotou, M. F. Briggs, \& G. J. Fishman (New York: AIP), 233

Rees, M. J., \& Mészáros, P., 1992, MNRAS, 258, 41P

Tavani, M. 1996, Phys. Rev. Letters, 76, 3478

van Paradijs, J., et al. 1997, Nature, 386, 686

von Montigny, C., et al. 1995, ApJ, 440, 525

Vietri, M. 1997, ApJ, 478, L9

Waxman, E. 1997, ApJ, 485, L5

Wijers, R. A. M. J., Mészáros, P., \& Rees, M. J. 1997, MNRAS, 288, L51 


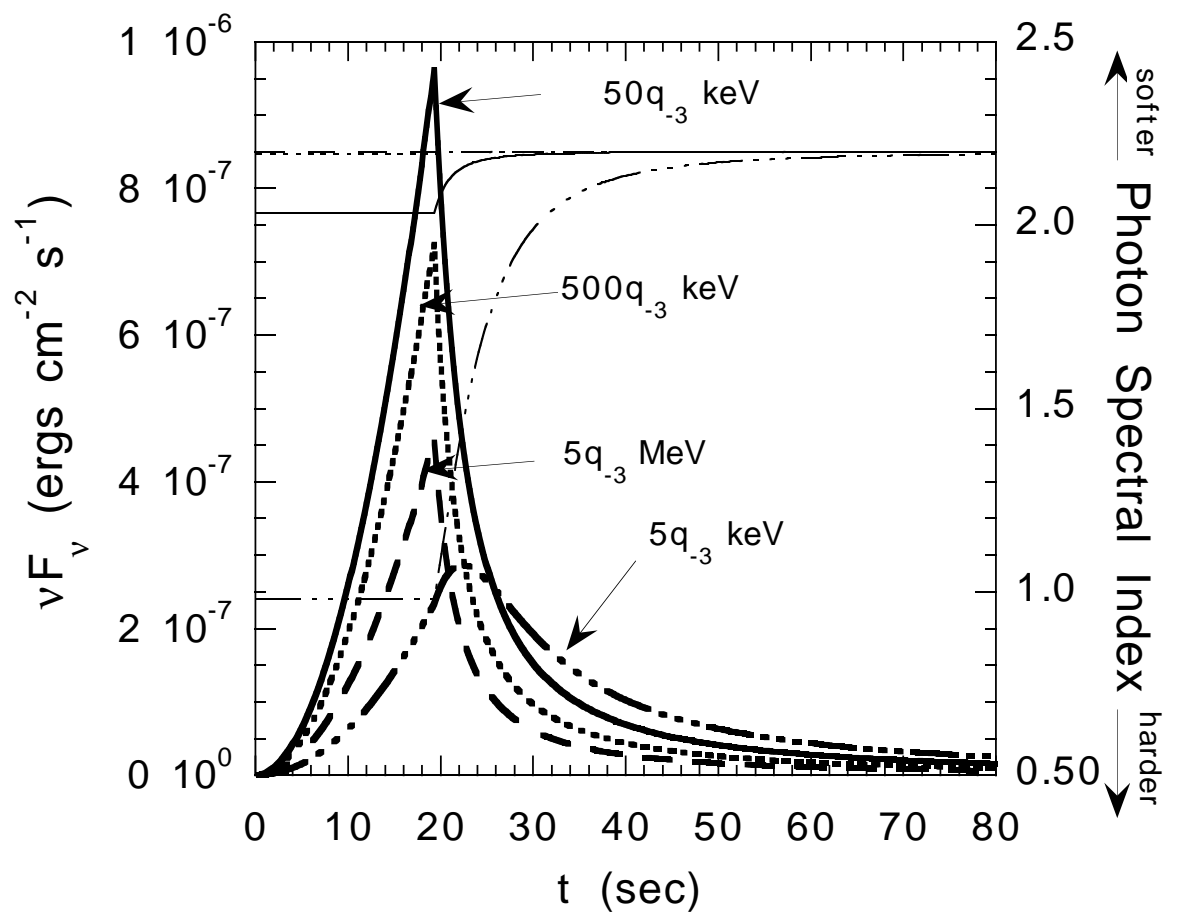

Fig. 1. - Light curves (thick lines) and spectral indices (thin lines) from a model GRB produced by a fireball with $\Gamma_{0}=300$ located at redshift $z=1$. Other parameters are listed in Table 1 . 


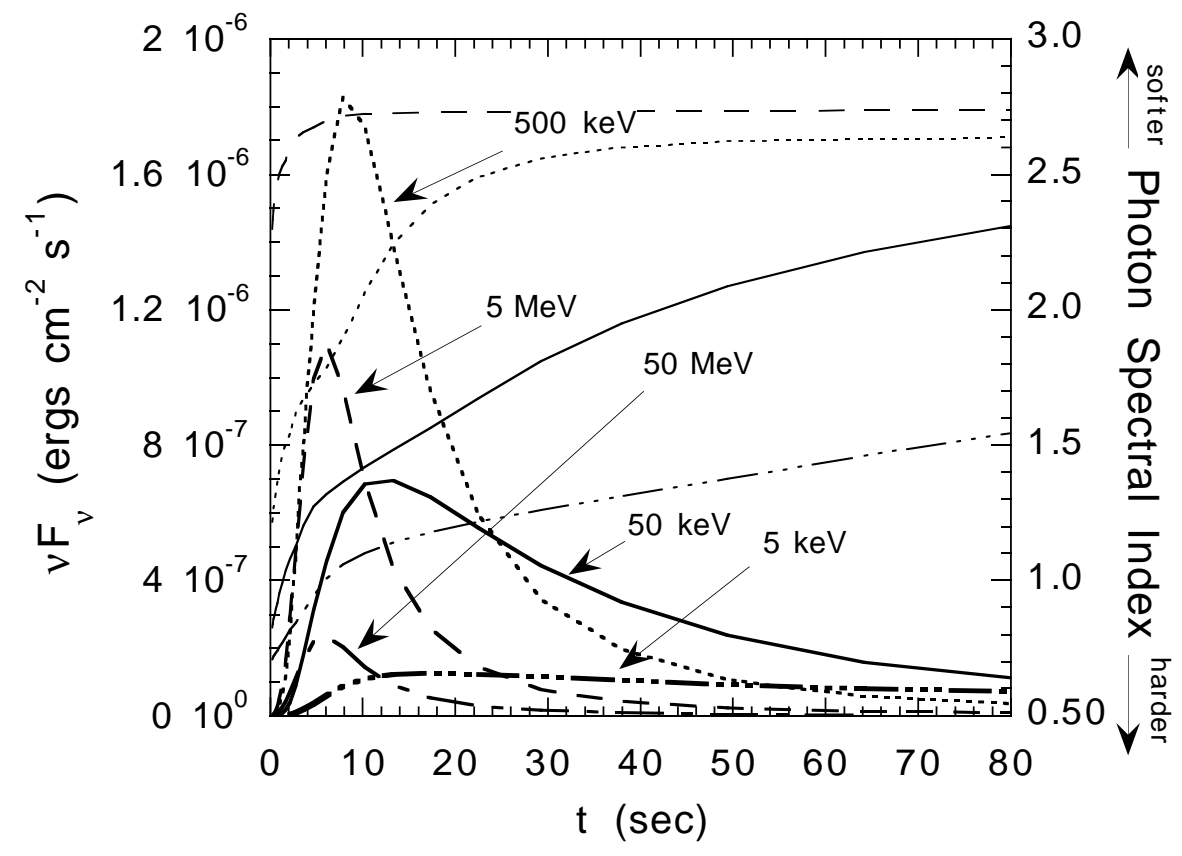

Fig. 2.- Numerical simulation results corresponding to the analytic model shown in Fig. 1 (see text for details). Equipartition between the energy in electrons, protons, and magnetic field is assumed. 